\title{
AVALIAÇÃO DO CARPROFENO E DO MELOXICAM COMO ANTIPIRÉTICOS EM CÃES
}

\author{
Avaliation of carprofen and meloxicam as antipyretics in dogs
}

\author{
Cláudia Turra Pimpão ${ }^{[a]}$, Francisco Pizzolato Montanha ${ }^{[b]}$, Carina Budziak ${ }^{[c]}$, \\ Luciana Ângelo Lima ${ }^{[\mathrm{d}]}$, Luiz Guilherme Achcar Capriglione ${ }^{[\mathrm{e}]}$, \\ Marina Figueiredo ${ }^{[\mathrm{f}]}$, Patrícia Mikos ${ }^{[\mathrm{g}]}$
}

\footnotetext{
[a] Doutora em Processos Biotecnológicos, Professora de Farmacologia e Toxicologia Veterinária na Pontifícia Universidade Católica do Paraná (PUCPR), Curitiba, PR -Brasil, e-mail: claudia.pimpao@pucpr.br

${ }^{[b]}$ Mestrando do Curso em Ciência Animal pela Pontifícia Universidade Católica do Paraná (PUCPR), Curitiba, PR -Brasil, e-mail: chicopm28@yahoo.com.br

${ }^{[c]}$ Mestrando do Curso em Ciência Animal pela Pontifícia Universidade Católica do Paraná (PUCPR), Curitiba, PR -Brasil, e-mail: c_budziak@yahoo.com.br

[d] Médica Veterinária-SESPO Vetbrands, Curitiba, PR-Brasil, e-mail: luciana.lima@ceva.com

${ }^{[e]}$ Residente da Pontifícia Universidade Católica do Paraná (PUCPR), Câmpus São José dos Pinhais, e-mail: luizcapriglione@yahoo.com.br

[f] Acadêmica do Curso de Medicina Veterinária pela Pontifícia Universidade Católica do Paraná (PUCPR), Curitiba, PR -Brasil, e-mail: marinaveterinaria@hotmail.com

[g] Acadêmica do Curso de Medicina Veterinária pela Pontifícia Universidade Católica do Paraná (PUCPR), Curitiba, PR -Brasil, e-mail:patriciaabreu@hotmail.com
}

\begin{abstract}
Resumo
Anti-inflamatórios não esteroidais (AINES) são utilizados como analgésicos e antipiréticos. São utilizados com frequência em animais como anti-inflamatório doméstico e fazem parte dos agentes terapêuticos mais utilizados de todos. O objetivo deste trabalho foi analisar a eficácia antipirética dos AINES carprofeno e meloxicam em cães. Foram utilizados 16 cães adultos, sem raça definida, divididos em quatro grupos (controle, dipirona, carprofeno e meloxicam). Todos os grupos receberam uma dose de lipopolissacarídeos (LPS) $(0,5 \mu \mathrm{g} / \mathrm{kg})$ para indução da temperatura febril e uma hora antes receberam dipirona $(25 \mathrm{mg} / \mathrm{kg})$, carprofeno $(4,4 \mathrm{mg} / \mathrm{kg})$ e meloxicam $(0,2 \mathrm{mg} / \mathrm{kg})$ por via oral. Antes e após a inoculação de LPS, as aferições clínicas foram realizadas a cada 15 minutos, até 180 minutos. Os animais foram observados por três dias consecutivos. Para a análise estatística dos dados foi utilizada o teste de Kruskal-Wallis. Os três grupos de cães que receberam dipirona, carprofeno e meloxicam tiveram redução significativa da febre $(p<0,01)$ em relação ao grupo controle (LPS). Não houve diferença significativa na frequência cardíaca nos quatro grupos analisados. Os cães que receberam dipirona e meloxicam apresentaram frequência respiratória menor do que o grupo que recebeu LPS $(\mathrm{p}<0,001)$, resultado este esperado por causa do controle eficaz da temperatura. A administração de LPS induz a um aumento
\end{abstract}


agudo da temperatura, portanto, conclui-se que o meloxicam, na dose de $0,2 \mathrm{mg} / \mathrm{kg}$, e carprofeno, na dose de $4,4 \mathrm{mg} / \mathrm{kg}$, por via oral, apresentam resposta antipirética, pois os dois ativos mantêm a temperatura próxima a $38,5^{\circ} \mathrm{C}$ por duas horas, como a dipirona.

Palavras-chave: Cães. Antipiréticos. Analgésicos não esteroidais.

\begin{abstract}
Non-steroidal anti-inflammatory drugs (NSAIDS) are used as analgesics and antipyretics. They are used frequently in domestic animals, besides anti-inflammatory being one of the most therapeutic agents used. The present research examined the effectiveness of antipyretic carprofen and meloxicam in dogs. Were used 16 adult dogs, mixed breed divided into four groups (control, dipyrone, carprofen and meloxicam). All groups received a dose of LPS $(0.5 \mu \mathrm{g} / \mathrm{kg})$ for induction of fever and one hour before the dogs received dipyrone (25 $\mathrm{mg} / \mathrm{kg})$, carprofen $(4.4 \mathrm{mg} / \mathrm{kg}$ ) and meloxicam $(0,2 \mathrm{mg} / \mathrm{kg})$ orally. Before and after inoculation of LPS the clinical measurements were taken every 15 to 180 minutes. The animals were observed for three consecutive days. For the statistical analysis of data was used the Kruskal-Wallis test. The three groups that received dipyrone, carprofen and meloxicam had a significant reduction of fever $(p<0.01)$ in the control group (LPS). There was no significant difference in heart rate in the four groups. The dogs that received meloxicam and dipyrone had lower respiratory rate than the group that received LPS $(p<0001)$, which was expected due to the effective control of temperature. Administration of LPS induces an acute increase in temperature, therefore it is concluded that meloxicam at a dose of $0.2 \mathrm{mg} / \mathrm{kg}$ and carprofen at a dose of $4.4 \mathrm{mg} / \mathrm{kg}$ orally, have antipyretic response, as two active keep the temperature close to $38.5^{\circ} \mathrm{C}$ for two hours as dipyrone.
\end{abstract}

Keywords: Dogs. Antipyretics. Non-steroidal analgesics.

\title{
INTRODUÇÃO
}

Além da atividade anti-inflamatória, os anti-inflamatórios não esteroidais (AINES) também são utilizados no combate à dor e à febre. O processo febril ocorre quando os leucócitos, que estão fagocitando partículas estranhas, liberam pirogênios endógenos, substâncias que se distribuem no organismo e promovem a liberação de prostaglandinas (PGE), principalmente a PGE2, que atuam sobre o hipotálamo, aumentando o limiar térmico. O mecanismo de ação principal da maioria dos AINES faz-se pela inibição da cicloxigenase (COX), a qual converte o ácido araquidônico em eicosanoides, mediadores responsáveis pela inflamação (LIVINGSTON 2000; RYN; PAIRET, 1999), inibindo a síntese de PGE e tromboxanos. Segundo Livingston (2000), a inibição da síntese de PGE pela enzima cicloxigenase responde, pelo menos em parte, por suas propriedades anti-inflamatória, analgésica e antipirética.

A febre é talvez o mais universal indicador de doenças e quando ocorre nos animais homeotérmicos, os mecanismos termorreguladores comportam-se como se tivessem ajustados para manter a temperatura corporal a um nível mais alto do que o normal, isto é, "como se o termostato tivesse sido reajustado" para um novo ponto acima de $37^{\circ} \mathrm{C}$ dependendo de cada espécie animal. Os receptores para temperatura então sinalizam que a temperatura vigente está abaixo do novo ponto fixo, e os mecanismos para aumentar a temperatura são ativados. Isto normalmente produz sensações de calafrio por causa da vasoconstrição cutânea e, ocasionalmente, tremor suficiente para produzir calafrios. Entretanto, a natureza da resposta depende da temperatura ambiente (BOOTH; McDONALD, 1992).

Toxinas bacterianas, como a endotoxina, atuam sobre monócitos, macrófagos e células de Kupffer para produzir as citocinas, que agem como pirógenos endógenos. As citocinas que produzem febre 
quando injetadas em animais experimentais incluem a IL-1, IL-6, vários interferons, lipopolissacarídeos (LPS) e TNF (Fator de Necrose Tumoral) (BOOTH; McDONALD, 1992; GANONG, 1998). Os lipopolissacarídeos (LPS), componentes celulares da parede de bactérias Gram-negativas podem atuar como endotoxinas e são determinantes na gênese de diversas patologias (GANONG, 1998).

Geralmente a resposta inflamatória local é iniciada e controlada pela intervenção de mediadores inflamatórios, entre os quais se destacam as PGE (LIVINGSTON, 2000). Segundo Taylor (1999), as PGE são sintetizadas a partir dos precursores localizados na membrana celular (fosfolipídios). O principal precursor é o ácido aracdônico, que serve de substrato para a enzima cicloxigenase (COX) para formar as PGE intermediárias $\left(\mathrm{PGG}_{2}\right.$ e $\left.\mathrm{PGH}_{2}\right)$. A partir delas são formados derivados mais estáveis $\left(\mathrm{PGE}_{2}, \mathrm{PGI}_{2}, \mathrm{PGF}_{2}\right)$ e tromboxanos.

Dor, inflamação e febre são frequentemente mediados, direta ou indiretamente, pela ação dos eicosanoides, incluindo prostaglandinas e tromboxanos (HAZEWINKEL et al., 2008).

A prostaglandina, por estimular os nociceptores, está associada à dor causada por injúria tecidual ou reação inflamatória. Há evidências de que os AINES também atuam no corno dorsal da medula espinhal, contribuindo para a ação analgésica e anti-inflamatória, sendo este efeito central, independente da inibição de COX (TAYLOR, 1999). Segundo Livingston (2000), a inibição da síntese de PGE pela enzima ciclooxigenase responde, pelo menos em parte, por suas propriedades antiinflamatória, analgésica e antipirética (LIVINGSTON, 2000). Pelo menos duas isoenzimas da cicloxigenase foram identificadas como determinantes de diferentes funções fisiológicas, COX-1 e COX-2 (GUÉRIUS et al., 2002). Os mediadores pró-inflamatórios, resultantes da ação da cicloxigenase1 (COX-1), que é a enzima constitutiva, estão representados pelas prostaglandinas relacionadas com os efeitos fisiológicos nos sistemas renal, gastrintestinal e cardiovascular (ALENCAR et al., 2003; BOVILL, 1997; FROLICH, 1997; GUÉRIUS et al., 2002; HAZEWINKEL et al., 2008; McCORMACK, 1994; NEIMI; TAXELL; ROSENBERG, 1997; VANE, 1995). Por outro lado, a cicloxigenase-2 (COX2), enzima induzida, leva à formação de PGE presentes no processo inflamatório (ALENCAR et al., 2003; CRYER; FELDMAN, 1998; GUÉRIUS et al., 2002; HAZEWINKEL et al., 2008; KAYMUGFORD et al., 2000). As diferenças entre os diversos AINES quanto à atividade anti-inflamatória e potencial ulcerogênico podem ser explicadas pela descoberta dessas duas isoformas da cicloxigenase, dessa forma, os AINES que bloqueiam inespecificamente as cicloxigenases predispõem o surgimento de efeitos colaterais, especialmente relacionados com o trato gastrintestinal (BOOTHE, 2001).

Os AINES têm sido utilizados com frequência em animais domésticos, por apresentarem efeitos antipiréticos, analgésicos e anti-inflamatórios e fazem parte dos agentes terapêuticos mais utilizados de todos (GUÉRIUS et al., 2002). Atualmente, há mais de cinquenta diferentes AINES no mercado e há, ainda, um fluxo contínuo de novas preparações. O grande número de novas substâncias significa que nenhuma dessas, até o momento, tem sido ideal no controle ou modificação dos sinais da inflamação, sem que haja efeitos deletérios no indivíduo (BOOTHE, 2001; BUDSBERG, 1999; FORSYTH; GUILFORD; HASLERTT, 1998; KORE, 1990). Desde a década de 90, os AINES vêm sendo introduzidos no mercado, com maior especificidade para COX-2, como, por exemplo: carprofeno, meloxicam, nimesulida, celocoxibe, etc. que têm diminuído drasticamente a incidência de efeitos colaterais (BUDSBERG, 1999; BUSCH et al., 1998; DOIG; PURBRICK; HARE, 2000; JUSTUS; QUIRKE, 1995; MACPHAIL, 2000; MATHEWS et al., 2001; PAIRET et al., 1998; RAINSFORD et al., 1999).

O meloxicam é um AINES desenvolvido em 1977 por Boehringer Ingelheim, na Germânia (TAKAHIRO et al., 2005), derivado do ácido enólico, relativamente novo, com propriedades analgésicas, anti-inflamatória e antipirética (JUSTUS; QUIRKE, 1995; SILVA et al., 2007). Também atua inibindo a infiltração de leucócitos no tecido inflamado, diminuindo a intensidade da agregação trombocitária induzida pelo colágeno, prevenindo assim a destruição que normalmente ocorre nos processos degenerativos osteoarticulares e músculo-esqueléticos (JUSTUS; QUIRKE, 1995). Desenvolve uma atividade inibitória seletiva sobre a COX-2 na cascata biossintética das prostaglandinas (ALENCAR; BUTERWORTH; WATERMAN, 2003). Reduz efeitos adversos como os gastrointestinais, tais como gastrites e úlceras (FROLICH, 1997; MONTOYA et al., 2004), e possui capacidade de inibir a inflamação na fase aguda (MARCHIONNI; PAGNONCELLI; REIS, 2006). 
O carprofeno é um agente anti-inflamatório com características analgésicas e antipiréticas. Em doses terapêuticas, possui pequeno efeito na inibição da síntese de PGE, mas é um potente inibidor da liberação dela (BENTON et al., 1997), com bom efeito anti-inflamatório e analgésico (NOLAN; REID, 1993; LASCELLES et al., 1994). O carprofeno é um acido carboxílico pertencente à classe dos AINES, conhecido como ácido aril-propiônico. O carprofeno é o fármaco mais lipofílico da sua classe e seu mecanismo de ação ocorre principalmente através da inibição da fosfolipase $\mathrm{A}_{2}$ e com pouca ação sobre as cicloxigenases. Por causa de sua inibição seletiva sobre a COX-2, o carprofeno provoca menor toxicose renal e gastrintestinal quando comparado a outras classes (GUÉRIUS et al., 2002). O grau de inibição da cicloxigenase promovido pelo carprofeno não permite prever por completo a eficácia clínica da droga. O bloqueio desta conversão resulta em menos ácido araquidônico disponível como substrato para a ação da cicloxigenase. Como resultado, menos PGE são produzidas, resultando em menos inflamação. Além disso, foi demonstrado que o carprofeno inibe a liberação de prostaglandinas em reações inflamatórias agudas e crônicas in vitro. O pico da concentração plasmática é 1 a 3 horas após a administração via oral (BOOTH; McDONALD, 1992; BUDSBERG, 1999; FORSYTH et al., 1998; FROLICH, 1997; SPINOSA; GÓRNIAK; BERNARDI, 2002).

O objetivo deste trabalho foi analisar a eficácia antipirética das drogas anti-inflamatórias não esteroidais do carprofeno e meloxicam em cães.

\section{MATERIAL E MÉTODOS}

Foram utilizados 16 cães adultos e sem raça definida (SRD) pertencentes ao Laboratório de Farmacologia e Toxicologia Veterinária da Pontifícia Universidade Católica do Paraná, provenientes do Centro de Controle de Zoonoses do Município de São José dos Pinhais, Estado do Paraná.

Este projeto foi avaliado e aprovado pelo Comitê de Ética no Uso de Animais da Pontifícia Universidade Católica do Paraná, sob parecer nº 221.

Todos os animais foram avaliados clinicamente antes, durante e após a experimentação, considerando os seguintes fatores: comportamento, temperatura, frequência cardíaca e frequência respiratória.

Para a indução da febre foi inoculado uma dose de lipopolissacarídeo (LPS) Escherichia coli 0111:B4 (Sigma, Chemical \& Co., EUA) diluído em salina estéril. A dose utilizada foi de 0,5 $\mu \mathrm{g} / \mathrm{kg}$ de LPS, por via intravenosa pré-determinada em experimentos anteriores no próprio laboratório de Farmacologia e Toxicologia Veterinária (PUCPR). Após a inoculação de LPS, as aferições clínicas foram realizadas a cada 15 minutos, encerrando em 180 minutos. Os animais foram observados por mais três dias consecutivos.

Os 16 animais foram divididos em quatro grupos contendo quatro animais cada: controle (C), dipirona (D), meloxicam (M) e carprofeno (CA). Uma hora antes da inoculação do LPS, os animais do grupo D receberam dipirona $(25 \mathrm{mg} / \mathrm{kg})$, os do grupo $\mathrm{M}$ receberam meloxicam $(0,2 \mathrm{mg} / \mathrm{kg})$ e os do grupo CA receberam carprofeno $(4,4 \mathrm{mg} / \mathrm{kg}$ ), todos por via oral. O intuito de usar o grupo D foi para ter um controle positivo, pois a dipirona é uma substância com comprovado efeito antitérmico.

Após 180 minutos da inoculação de LPS, os animais que apresentavam temperatura elevada receberam uma dose de dipirona e os que apresentaram vômito receberam uma dose de metaclopramida $(0,3 \mathrm{mg} / \mathrm{kg}$,via SC).

Para a análise estatística dos dados foi utilizado o teste de Kruskal-Wallis, caso encontradas diferenças significativas ( $\mathrm{p}<0,05)$, o teste de comparações Dunn's foi aplicado. O nível de significância adotado foi $5 \%(\alpha=0,05)$. Todos os cálculos foram realizados utilizando o Software estatístico GraphPad Prism version 3.00 for Windows, San Diego - Califórnia, EUA.

\section{RESULTADOS}

O LPS estimula o vômito em cães, por causa do aumento da temperatura e dor deixa o animal muito apático, sendo esperado que os animais que receberam somente LPS apresentassem vômito e 
apatia. Ante esses efeitos, todos os animais receberam uma dose de dipirona após 180 minutos da inoculação do LPS e os que apresentaram vômito receberam uma dose de metaclopramida. Dentre as alterações endócrinas-metabólicas decorrentes do estresse, como a inoculação do LPS, destacam-se a liberação de catecolaminas e cortisol, com consequente aumento do metabolismo basal, do trabalho cardíaco, da resistência vascular periférica e do consumo de oxigênio pelo miocárdio (SAKATA, 2001). A dor pode ocasionar inapetência, emagrecimento e alterações de comportamento (HASKINS, 1992).

Os três grupos de cães que receberam dipirona, carprofeno e meloxicam tiveram redução significativa da temperatura febril $(\mathrm{p}<0,01)$ em relação ao grupo controle (LPS) (Figura 1). O Grupo controle apresentou aumento da temperatura gradativo até os 180 minutos de aferição, chegando em torno de $40 \mathrm{C}$, sendo que os outros três grupos mantiveram-se em torno 38,5 a $38,9^{\circ} \mathrm{C}$, não ultrapassando $39^{\circ} \mathrm{C}$. Os animais que receberam dipirona mostraram-se mais estáveis em relação à temperatura, não ultrapassando $38,5^{\circ} \mathrm{C}$, apresentando diferença significativa em relação aos cães que receberam carprofeno e meloxicam (Figura 1). Não houve diferença entre as temperaturas dos cães que receberam carprofeno e meloxicam.

A dipirona sódica é um derivado pirazolônico hidrossolúvel que foi introduzido para uso clínico na Alemanha, em 1922, como analgésico, antitérmico e antiespasmódico (LEVY; ZYLBERKATZ, 1995). Em 2001, a Agência Nacional de Vigilância Sanitária (ANVISA), juntamente com autoridades nacionais e internacionais, confirmam que a eficácia da atividade antipirética e analgésica da dipirona é inquestionável, sendo o medicamento desta classe mais utilizado no Brasil (ANDRADE, 2001).

De acordo com os resultados obtidos, observamos que até 2 horas após a administração do LPS os três medicamentos (dipirona, carprofeno e meloxicam) conseguiram manter a temperatura febril, após esse período, a temperatura tende a elevar-se. Já o grupo que recebeu somente LPS, manteve a temperatura febril durante todo o experimento, como mostra a Figura 1.

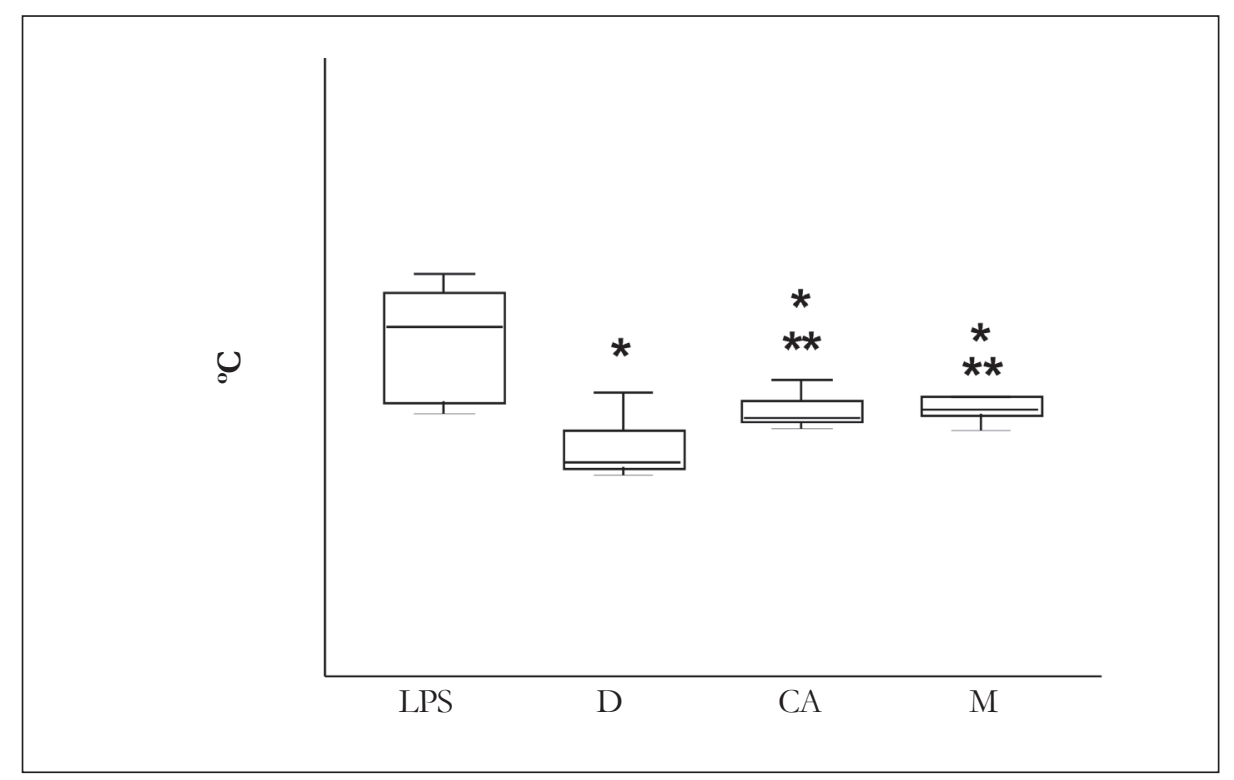

FIGURA 1 - Comparação dos valores de temperatura retal dos cães inoculados com lipopolissacarídeo (LPS) e receberam dipirona (D), carprofeno (CA) e meloxicam (M)

Nota: $* \mathrm{p}<0,001$ em relação ao grupo que recebeu LPS. ${ }^{* *} \mathrm{p}<0,01$ em relação ao grupo que recebeu dipirona.

Não houve diferença significativa entre a frequência cardíaca nos quatro grupos analisados, contudo, houve grande variação nos batimentos cardíacos dos animais que receberam somente LPS e também nos do grupo que recebeu o carprofeno (Figura 2). 


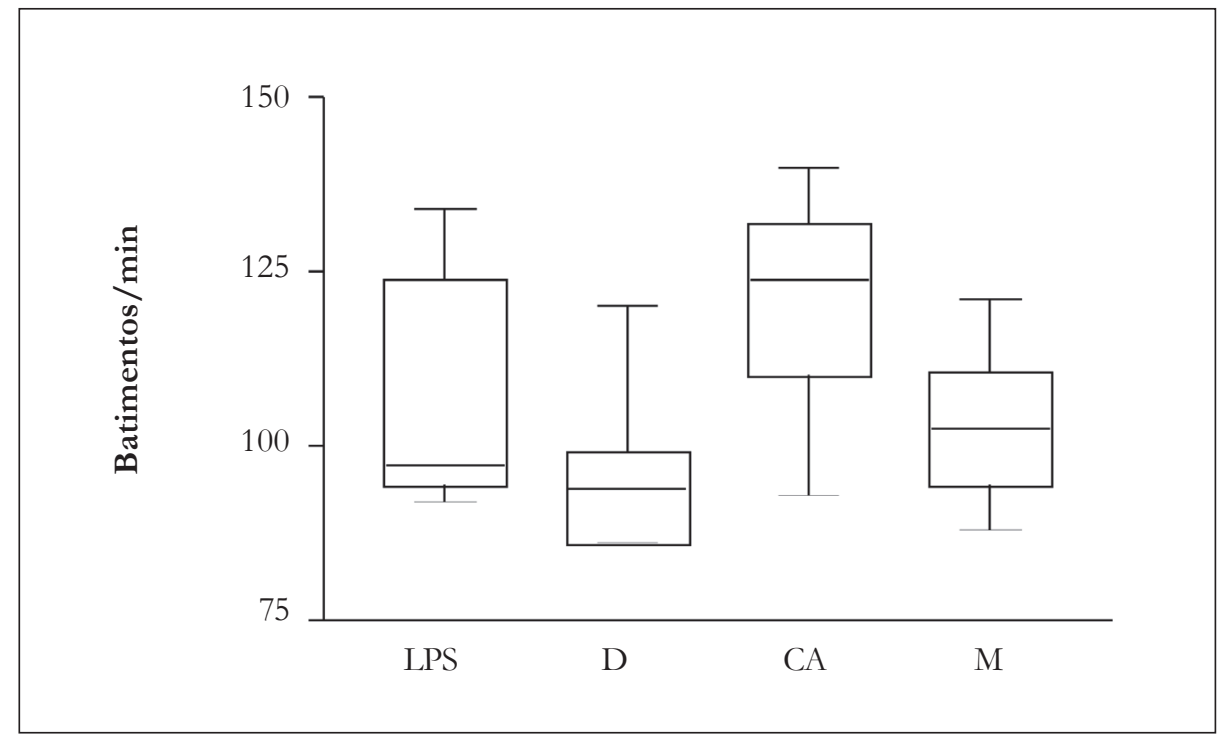

FIGURA 2 - Comparação dos valores de frequência cardíaca dos cães inoculados com lipopolissacarídeo (LPS) e receberam dipirona (D), carprofeno (CA) e meloxicam (M)

Os cães que receberam dipirona e meloxicam apresentaram frequência respiratória menor do que o grupo que recebeu somente LPS ( $p<0,001$ ), representado na Figura 3, resultado este esperado por causa do controle eficaz da temperatura. Porém os animais que receberam carprofeno não apresentaram diferença em relação ao grupo controle mesmo apresentado o controle da temperatura. Nos cães a sudorese é insignificante na termorregulação, sendo que o ofego é mais importante, pois é um importante mecanismo regulador da temperatura em muitas espécies, particularmente em cães. A frequência respiratória pode aumentar para 200 a 400 respirações por minuto, e o volume corrente diminui durante os ofegos, de tal modo que a ventilação alveolar permanece constante (SWENSON; REECE, 1996).

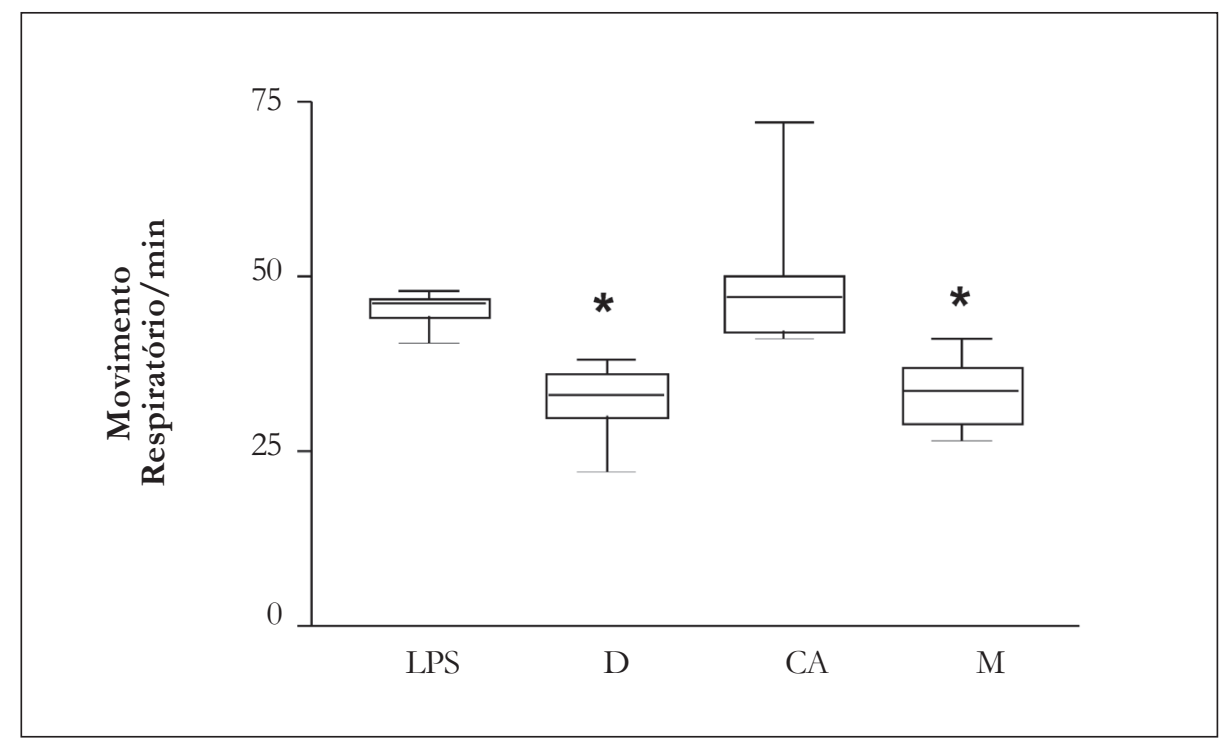

FIGURA 3 - Comparação dos valores de frequência respiratória dos cães que foram inoculados com lipopolissacarídeo (LPS) e receberam dipirona (D), carprofeno (CA) e meloxicam (M)

Nota: ${ }^{*} \mathrm{p}<0,001$ em relação ao grupo que recebeu LPS. 
Todos os animais foram observados por mais três dias e todos estes apresentaram comportamento normal.

\section{CONCLUSÃO}

A administração de LPS induz a um aumento agudo da temperatura, portanto, pode-se concluir que o meloxicam, na dose de $0,2 \mathrm{mg} / \mathrm{kg}$, e o carprofeno, na dose de $4,4 \mathrm{mg} / \mathrm{kg}$, administrados via oral, apresentam uma resposta antipirética, pois esses dois princípios ativos mantêm a temperatura em torno de $38,5^{\circ} \mathrm{C}$ por duas horas, como a dipirona.

\section{REFERÊNCIAS}

ALENCAR, M. M. A. et al. Margem de segurança do meloxicam em cães: efeitos deletérios nas células sanguíneas e trato gastrintestinal. Ciência Rural, Santa Maria, v. 33, n. 3, p. 525-532, 2003.

ANDRADE, S. F. et al. Estudo clínico e histopatológico do uso de antiinflamatórios inibidor seletivo COX-2 (meloxicam) em cães. A Hora Veterinária, Porto Alegre, ano 20, n. 120, p. 44-46, 2001.

BENTON, H. P. et al. Effect off carprofen on sulfated glycosaminoglycan metabolism, protein syntesis, and protaglandin realese by cultered ostearthritic canine chondrocyrtes. American Journal of Veterinary Research, Schaumburg, v. 3, n. 58, p. 286-92, 1997.

BOOTHE, D. M. Anti-inflammatory drugs. In: BOOTHE, D.M. Small animal clinical pharmacology and therapeutics. Philadelphia: Saunders, 2001. Cap. 16, p. 281-311.

BOOTH, N. H.; McDONALD, L. E. Farmacologia e terapêutica em veterinária. 8. ed. Rio de Janeiro: Guanabara Koogan, 1992.

BOVILL, J. G. Mechanisms of actions of opiods and nonsteroidal anti-inflamatory drugs. European Journal of Anaesthesiology, Cambridge, n. 14, p. 9-15, 1997. Suplemento 1.

BUDSBERG, S. C. Tendencias actuales y futuras en el uso de los AINEs para el tratamiento de la osteoartritis en los perros. Waltham Focus, Hannover, v. 9, n. 2, p. 26-31, 1999.

BUSCH, U. et al. Kinetics of meloxicam in animals and the relevance to humans. Drug Metabolism and Disposition, Stanford, v. 26, n. 6, p. 576-584, 1998.

CRYER, B.; FELDMAN, M. Cyclooxygenase-1 and ciclooxygenase-2 selectivity of widely used nonesteroidal anti-inflamatory drugs. American Journal of Medicine, Arizona, v. 104, n. 5, p. 413-421, 1998.

DOIG, P. A.; PURBRICK, K. A.; HARE, J. E. Clinical efficacy and tolerance of meloxicam in dogs with chronic osteoarthritis. Canadian Veterinary Journal, Ottawa, v. 41, n. 4, p. 296-300, 2000.

FORSYTH, S. F.; GUILFORD, W. G.; HASLETT, S. J. Endoscopy of the gastroduodenal mucosa after carprofen, meloxicam and ketoprofen administration in dogs. Journal of Small Animal Practice, Gloucester, v. 39, n. 9, p. 421-424, 1998.

FROLICH, J. C. A classification of NSAIDs according to the relative inhibition of cyclooxygenase isoenzymes. Trends in Pharmacological Sciences, New York, v. 18, p. 30-34, 1997.

GANONG, W. F. Fisiologia médica. 17. ed. Rio de Janeiro: Guanabara Koogan, 1998c.

GUÉRIUS, S. D. et al. Exame gastroscópico após administração oral de anti-inflamatórios não esteroidais em cães. Archives of Veterinary Science, Curitiba, v. 7, n. 2, p. 53-58, 2002. 
HASKINS, H. C. Postoperative analgesia. Journal of Small Animal Practice, Gloucester, v. 22, n. 2, p. 353-366, 1992.

HAZEWINKEL, H. A. W. et al. Comparison of the effects of firocoxib, carprofen and vedaprofen in a sodium urate crystal induced synovitis model of arthritis in dogs. Research in Veterinary Science, New York, v. 19, n. 6, p. 74-79, 2008.

JUSTUS, C.; QUIRKE, J. F. Dose-response relationship for antipyretic effect of meloxicam in an endotoxin model in cats. Veterinary Research Communications, Edinburgh, v. 19, n. 4, p. 312 330, 1995.

KAY-MUGFORD, P. et al. In vitro effects of nonsteroidal anti-inflammatory drugs on cyclooxygenase activity in dogs. American Journal of Veterinary Research, Schaumburg, v. 61, n. 7, p. 802-810, 2000.

KORE, A. M. Toxicology of nosnsteroidal antiinfflammatory drugs. Journal of Small Animal Practice, Gloucester, v. 20, n. 2, p. 419-430, 1990.

LASCELLES, B. D. X.; BUTERWORTH, S. J.; WATERMAN, A. E. Postoperative analgesic and sedative effects of carprofen and pethidine in dogs. Veterinary Record, London, v. 134, n. 8, p. 187-191, 1994.

LEVY, M.; ZYLBER-KATZ, E.; ROSENKRANZ, B. Clinical pharmacokinetics of dypirone and its metabolites. Clinical Pharmacokinetics, Auckland, v. 28, n. 3, p. 213-216, 1995.

LIVINGSTON, A. Mechanism of nonsteroidal anti-inflamatory drugs. Journal of Small Animal Practice, Gloucester, v. 30, p. 773-782, 2000.

MACPHAIL, C. M. Tratamiento de la osteoartritis canina. Waltham Focus, Hannover, v. 10, n. 1, p. 25-31, 2000.

MARCHIONNI, A. M. T.; PAGNONCELLI, R. M.; REIS, S. R. A. Influência do meloxicam e da dexametasona no processo inflamatório e no reparo tecidual. Revista Odonto Ciência, Porto Alegre, v. 21, n. 51, p. 23-28, 2006.

MATHEWS, K. A. et al. Safety and efficacy of preoperative administration of meloxicam, compared with that of ketoprofen and butorphanol in dogs undergoing abdominal surgery. American Journal of Veterinary Research, Schaumburg, v. 62, n. 6, p. 882-888, 2001.

McCORMACK, K. Non-steroidal anti-inflammatory drug and spinal nociceptive processing. Pain, Edinburgh, v. 59, n. 1, p. 9-43, 1994.

MONTOYA, L. et al. A pharmacokinetic comparison of meloxicam and ketoprofen following oral administration to healthy dogs. Veterinary Research Communications, Dordrecht, v. 28. n. 5, p. 415-428, 2004.

NEIMI, T. T.; TAXELL, C.; ROSENBERG, P. H. Comparison of effect of intravenous ketoprofen, ketorolac and diclofenac on platelet function in volunteers. Acta Anaesthesiologica Scandinavica, Copenhagen, v. 41, n. 10, p. 1353-1358, 1997.

NOLAN, A.; REID, J. Comparison of the postoperative analgesic and sedative effects of carprofen and papaveretum in the dog. Veterinary Research Communications, Dordrecht, v. 133, n. 10, p. 240-242, 1993.

PAIRET, M. et al. Differential inhibition of cyclooxygenases 1 and 2 by meloxican and its 4 '-isomer. Inflammation Research, Birkhäuser, v. 47, n. 6, p. 270-276, 1998. 
RAINSFORD, K. D. et al. Effects of the NSAIDs meloxicam and indomethacin on cartilage proteoglycan synthesis and joint responses to calcium pyrophosphate crystals in dogs. Veterinary Research Communications, Dordrecht, v. 23, n. 2, p. 101-113, 1999.

RYN, J. V.; PAIRET, J. Inflamation and NSAIDS: Cox 1 and Cox 2 and preferential Cox-2 inhibition. In: Recent advances in non-steroidal anti-inflamatory therapy in small animals. Paris: Boehringer Ingelheim, 1999. p. 11-13.

SAKATA, R. K. Tratamento da dor. In: YAMASHITA, A. M. et al. Anestesiologia/SAESP. 5. ed. São Paulo: Atheneu, 2001. p. 1165-1182.

SILVA, J. G. et al. Estudos preliminares do uso do meloxicam em gatas: analgesia e influência sobre uréia e creatinina sérica. A Hora Veterinária, Porto Alegre, ano 26, n. 155, p. 17-18, 2007.

SPINOSA, H. S.; GÓRNIAK, S. L.; BERNARDI, M. M. Farmacologia aplicada à medicina veterinária. 3. ed. Rio de Janeiro: Guanabara Koogan, 2002.

SWENSON, M. J.; REECE, W. O. Fisiologia dos animais domésticos. 11. ed. Rio de Janeiro: Guanabara Koogan, 1996.

TAKAHIRO, N. et al. Meloxicam inhibits osteosarcoma growth, invasiveness and metastasis by COX-2-dependent and independent routes. Carcinogenesis, Oxford, v. 27, n. 3, p. 410-414, 2005.

TAYLOR, P. Newer analgesics. Journal of Small Animal Practice, Gloucester, v. 29, n. 3, p. 719 735, 1999.

VANE, J. R. NSAIDs, COX-2 inhibitoris, and the gut. The Lancet, London, v. 346, n. 8982, p. 1105-1106, 1995.

Recebido: 04/10/2008

Received: 10/04/2008

Aprovado: 20/03/2009

Approved: 03/20/2009 